\title{
HUMAN ROTAVIRUS GROUP A SEROTYPES CAUSING GASTROENTERITIS IN CHILDREN LESS THAN 5 YEARS AND HIV-INFECTED ADULTS IN VIWANDANI SLUM, NAIROBI
}

\author{
Raini SK ${ }^{1}$, Nyangao $\mathbf{J}^{2}$, Kombich $\mathbf{J}^{2}$, Sang' $\mathbf{C}^{1}$, Gikonyo $\mathbf{J}^{2}$, Ongus $\mathbf{J R}^{1}$, Odari \\ EO $\mathbf{O}^{1,3}$
}

\begin{abstract}
BACKGROUND: Rotavirus remains a leading cause of acute gastroenteritis in children worldwide with an estimated 2000 deaths each day in developing countries. Due to HIVIAIDS scourge in Kenya, it is possible that rotavirus-related gastroenteritis has been aggravated in adults. The Global Alliance for Immunizations has ranked rotavirus infection a priority for vaccine, and, to ensure its success, there is a need to document the local strain(s) circulating in different regions.

METHODS: A cross-sectional study was conducted to document human rotavirus group A serotypes in children below 5 years and HIV-infected adults in Viwandani slum in Nairobi, Kenya. A total of 260 (128 from children and 132 from HIV infected adults) fecal specimen samples were analyzed from August 2012 to July 2013. Screening for rotavirus was done by antigen based enzyme immune-sorbent assay (ELISA), Polyacrylamide gel electrophoresis (PAGE) was used to detect rotavirus electropherotypes and finally genotyping was done by RT-PCR using genotype-specific primer sets targeting VP4 and VP7 genes.

RESULTS: Rotavirus was detected in $23 \%$ and $8 \%$ of children and adult, respectively. Prevalence was high in children of $<2$ years and adults of $>48$ years. Long electropherotypes accounted for $80 \%$ and 60\% while short electropherotypes accounted for $20 \%$ and $40 \%$ in children and adult, respectively. The common globally distributed strains, G1 and G3, accounted for $60 \%$ detections while the unusual G9 strain accounted for $80 \%$ infection in adults. G1P[8] was the common genotypic combination in children, accounting for $40 \%$ infection, whereas $G 9$ [P8] accounted for $60 \%$ of the infections in adults.

CONCLUSION: This study shows the existence of strain diversity between rotavirus circulating in children and adults within this study group. It further shows that as currently constituted, the 2 vaccines recommended for rotavirus would cover the circulating strain in Viwandani slum. Finally, there is a need for continuous rotavirus strain surveillance in children and a further focus on HIV infected adults.

KEYWORDS: Rotavirus, Diarrhoea, Gastroenteritis, Viwandani slum, Kenya
\end{abstract}

DOI: http://dx.doi.org/10.4314/ejhs.v25i1.6

\section{INTRODUCTION}

Severe diarrhea due to rotavirus infection remains a major public health concern in Sub-Saharan Africa. Globally, it is estimated that rotavirus causes approximately 527,000 deaths per year; almost half of these deaths $(230,000)$ occur in
Sub-Saharan Africa (1). In a study covering 10 countries in Sub-Saharan Africa, it was observed that $>40 \%$ of all episodes of acute gastroenteritis affecting children under 5 years occur due to rotavirus infection (2). The same study observed that the burden of the disease was mainly

\footnotetext{
${ }^{1}$ College of Health Sciences, Jomo Kenyatta University of Agriculture and Technology, Nairobi, Kenya

${ }^{2}$ Centre for Virus Research, Kenya Medical Research Institute, Nairobi, Kenya

${ }^{3}$ Max von Pettenkofer-Institute, Munich, Germany

Corresponding Author: Odari EO, Email: Kodarie04@yahoo.com
} 
experienced in the health sector where $>40 \%$ of hospital admissions were due to rotavirus infections.

In 2008, the World Health Organization (WHO) also reported rotavirus as the most common cause of severe diarrheal disease hospitalizations in young children in Africa (3). In fact, some studies carried out in Kenya have shown prevalence as high as $29 \%$ of rotavirus infections in hospitalized patients (4) compared to only $18 \%$ of outpatient cases (5). These findings of high burden of rotavirus in hospitalized children puts pressure not only on the limited bed capacity in many African nations but also on the economy, since women who contribute significantly to the economies would therefore spend a lot of time in hospitals with children away from work. Furthermore, the affected children also end up wasting a lot of time away from schools.

Contrary to the belief of rotavirus infections only in children, some studies have reported infections in adults (6-9). Routes of transmission in adults have also been described (10) as including transmission from children to adults which has come out significantly in a majority of studies (11-13). With the high level of poverty and the effects of HIV/AIDS scourge in many SubSaharan African countries, an increased burden of rotavirus infection would be expected especially in immunocompromised populations.

Currently, the control of rotavirus in adults, especially in the immunocompromised remains a great challenge; however, it is expected that the vaccines introduced $(14,15)$ for prevention of rotavirus infection in children, if successful, could provide indirect protection of these adults (16). However, even with the efficacy of the approved vaccines ranging from 89 to $98 \%$ in the industrialized world and parts of Latin America (17-20), a reduced efficacy of between 39 to $77 \%$ has been shown for the developing countries in Africa and Asia (21-23). Although the difference in efficacy between the developed and the developing world was mainly attributed to hostrelated factors, (22) strain diversity still remains a fundamental factor especially in ensuring efficacy of vaccines in this part of the world. This study therefore aimed to document the strains of rotavirus circulating within a poor urban setting in Nairobi, Kenya, and to compare these strains between children and adults.

\section{MATERIALS AND METHODS}

Study Setting: This study was conducted in 2 health facilities within Viwandani slum in Nairobi. This slum is located about $7 \mathrm{kms}$ from Nairobi City Centre and extends into the Nairobi's industrial area. The population here is mainly comprised of casual labourers working within the industrial area. The slum is characterized by poor sanitation and waste disposal. Apart from malnutrition and HIV/AIDS, other common public health challenges include diarrhoea, cholera and typhoid fever.

Study population and sample material: Since rotavirus infection is self-limiting, stool samples were collected only from patients who complained of gastroenteritis for not more than 5 days. Upon receipt, the samples were transported, tested and analysed at the Centre for Virus Research, Kenya Medical Research Institute in Nairobi. Included in the study were children $<5$ years old and HIV antibody positive adults. A total of 260 samples were collected and tested. Out of these, 128 specimens were collected from children and 132 from adults.

Specimen processing: Upon receipt, samples were tested by an antigen based Enzyme Linked Immunosorbent Assay (ELISA) and positive samples subjected to Poly Acrylamide Gel Electrophoresis (PAGE) to determine rotavirus electropherotypes. Further analysis of the samples was done in a nested RT-PCR as had been previously described (24). In the first reaction, VP4 and VP4 target specific primers TGGCTTCGCCATTTTATAGACA (position 1132) and ATTTCGGACCATTTATAACC (position 868-887) were used.

In the second reaction, primer ATTTCGGACCATTTATAACC (position 868887) and a mixture of primers targeting specific variable regions of the VP4 and VP7, namely; the G1, G2, G3, G4, G6, G8, G9 and P4, P6, P8, P9 and P10 were used.

Ethical Clearance: This study was approved by the ethical committee of the Kenya Medical Research Institute. Permission to use samples from the 2 health institutions was also granted by the Health Department of the Nairobi City Council.

Data analysis: Data were entered and analyzed in SPSS ver.16. Chi square test was performed to 
determine the difference in distribution patterns of rotavirus infection. $\mathrm{P}<0.05$ was considered significant.

\section{RESULTS}

A total of $30(23 \%)$ specimens from children and $10(8 \%)$ (Table 1) from adults were detected positive by ELISA. Analysis of detections in children $<5$ years showed that the highest prevalence was recorded within the age group of 7 to 12 months followed by those within the age group of 13 to 24 months. The least prevalence was recorded for the group of $<1$ to 3 months (Figure 1). In the HIV antibody positive adults (Figure 2), rotavirus infection was realized in high frequency in the group of 49 to 58 years. The results showed no difference in distribution of rotavirus infection in the 2 groups based on gender.

Table 1: Distribution of rotavirus infection in adults and children $(n=260)$

\begin{tabular}{lllll}
\hline \multirow{2}{*}{ Patient category } & \multicolumn{2}{l}{ Outcome } & P* \\
\cline { 3 - 5 } & & Positive & Negative & \\
\hline Adults & 10 & 122 & 0.0002 \\
Children & & 30 & 98 & \\
\hline Adults & Male & 3 & 42 & 1.0000 \\
& Females & 7 & 80 & \\
\cline { 2 - 5 } Children & Male & 14 & 50 & 0.8350 \\
& Females & 16 & 48 & \\
\hline
\end{tabular}

$*$ value $<0.05$ considered significant

\section{Age of rotavirus infection in children $(n=30)$}

\section{Frequency of rotavirus infection in adults $(n=10)$}

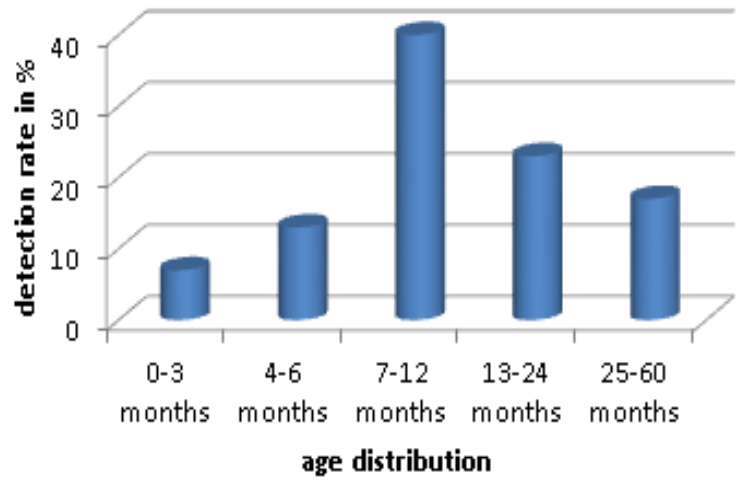

Fig 1: Shows the age distribution of children $<5$ years. Highest frequency was realized for children between 6 months to 2 years, which could be attributed to the development stage of the children when most of them get exposed to a high risk of fecal-oral infections.

Analysis of electropherotypes revealed that $80 \%$ (24) of the isolated strains were long electropherotypes while $20 \%$ (6) of the strains were short electropherotypes among children. Long electropherotypes accounted for 60\% (6) and short accounted for $40 \%$ (4) in adults. No mixed

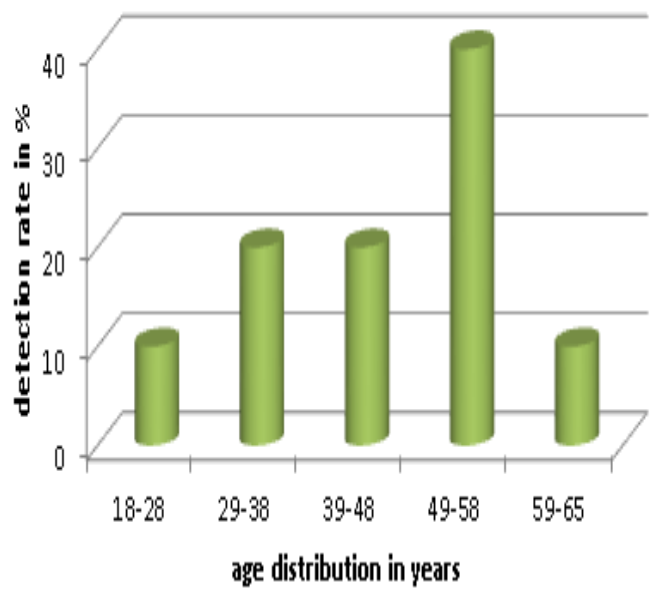

Fig 2: Frequency of rotavirus infection in adults $\{\mathrm{n}=10\}$

infection was detected within the electropherotypes. Long electropherotypes were therefore predominant during this study.

It was also realized that the long electropherotypes did not appear to vary with age among children and occurred in all ages (one month to five years old). Short electropherotypes 
on the other hand were identified only in children $<2$ years old.

A total of 27 (90\%) samples were genotyped for VP7 associated types among the 30 PAGE positive samples from children. Results showed that G1 accounted for $47 \%$ (14), followed by G9 $30 \%$ (9) and G3, 13\% (4). One sample (3.7\%) was a mixed type (G3/G9), while the remaining 2 samples, although positive by ELISA and PAGE, could not be sufficiently typed. Other genotypes including G2, G4 and G8-type viruses were not detected among this group of samples. On the other hand, results for VP4 associated types showed that $25(83.3 \%)$ of the 30 PAGE positive samples from children could sufficiently be typed. Only $2 \mathrm{P}$ types were shown to be in circulation with $\mathrm{P}[6]$ accounting for $6.7 \%$ (2) and $\mathrm{P}[8]$ accounting for $77 \%$ (23). Two (6.7\%) samples had mixed (P[6]/P[8]) strains while $3(10 \%)$ could not efficiently be typed. P [9] or P[10] type viruses were not detected.

Throughout the study, the long electropherotypes were always associated with G1 and G9 genotypes, whereas the short electropherotypes were associated with genotype G3.

During the typing of assays, it was observed that a specific $G$ type could always be correlated to a P type; namely, G1, and G3 always coexisted with P[8], while G9 was found to associate with $\mathrm{P}[6]$ or P[8]. Overall, G1 P[8] was recorded as the most common in children accounting for $44 \%$ of infections while G9[P6] at $40 \%$ was the most common in adults (Table 2).

Table 2: Prevalence of rotavirus genotypes detected in adults and children $(n=40)$

\begin{tabular}{llll}
\hline G-P type combination & $\begin{array}{l}\text { Children } \\
(\mathbf{N}=\mathbf{3 0})(\%)\end{array}$ & $\begin{array}{l}\text { Adults } \\
(\mathbf{N}=\mathbf{1 0})(\%)\end{array}$ & $\begin{array}{l}\text { Total } \\
(\mathbf{N}=(40)(\%)\end{array}$ \\
\hline Common types & & & $11(27.5)$ \\
G1P[8] & $11(37)$ & 0 & $9(22.5)$ \\
G9P[8] & $5(16)$ & $4(40)$ & $9(22.5)$ \\
G3P[8] & $6(20)$ & $3(30)$ & $5(12.5)$ \\
\hline Uncommon types & & & 0 \\
G9P[6] & $3(10)$ & $2(20)$ & $2(5)$ \\
G1P[4] & 0 & 0 & \\
Mixed infection & $2(7)$ & 0 & 0 \\
\hline Non typeable & & & $1(2.5)$ \\
G* - P** & 0 & 0 & $3(7.5)$ \\
G** - P* & $1(3)$ & 0 & \\
G* - P* & $2(7)$ & $1(10)$ & \\
\hline$*$ non typeable & $* *$ positive & &
\end{tabular}

\section{DISCUSSION}

This study reports a prevalence of $23 \%$ and $8 \%$ of rotavirus infection in children below 5 years and HIV infected adults, respectively. It further reports a prevalence of $40 \%$ circulation of G1P[8] in children and $60 \%$ circulation of G9P[8] in adults.

The findings regarding rotavirus prevalence in children reflects previous results which reported rotavirus in $18 \%$ in outpatient setting (5) and $30 \%$ in an inpatient setting (4). The burden of rotavirus infection in children below 5 years in Africa has been well elucidated in other studies (2). In our study within this age group, rotavirus infection was most prevalent in children in between 7-12 months, only equivalent to those between 13-24 and 25-60 months combined. Overall, children under 1 year contributed to over $17 / 30(57 \%)$ of positive cases, and over $24 / 30(80 \%)$ of them had rotavirus diarrhoea at the age of 2 years. This finding is a direct contradiction to the findings of Nokes and colleagues (4) which found a higher prevalence among children aged 3-5 years. The difference may be attributed mainly to the settings 
of the studies. The age group with the high prevalence of infection in our study was comprised of children either crawling or just learning to walk and as such would have a high risk for fecal oral transmission as they eat anything from the ground or suckle their fingers. From our observation of the conditions in Viwandani slum, it is unlikely that the care-givers spend time with the children (since a lot of time is taken for casual jobs in the neighboring up-market estates). As a result children are left under the care of the neighbors or underage female relatives. Even within the facility, it is more likely to test only children above 3 years for gastroenteritis related pathogens compared to those below 2 years since those below this age normally undergo empiric management (based on clinical symptoms) and rehydration therapy using the oral rehydration salts. This could also explain the results of Noke's study where 224 cases were tested for the group between 3-5 years compared to only 84 cases tested for the group between $0-2$ years. In their work, Temu and colleagues (25) also showed high prevalence of rotavirus infection among children below 2 years in Tanzania. As had been anticipated, there was no significant difference in the rate of infection between male and female children.

To the best of our knowledge, there is currently very little or no information on the prevalence of rotavirus infection among Kenyan adults, and as such, we are unable to relate our findings within this population to any similar study in Kenya. Although, our study in adults was carried out in HIV antibody positive adults. The data presented here did not evaluate among other things the immune status (through CD4 counts) or the viral load of the patients prior to collection of samples for analysis. Therefore, as such we cannot conclusively show that infection by rotavirus was as a result of AIDS disease. Further, we also note that although the highest prevalence within this group was between the age of $48-59$ years (50\% of the total adult cases), the data $(n=10)$ was also very small for conclusive evaluation of low immune status within this group. We can, however, hypothesize that the majority of women in poor settings in Kenya within this age group, currently remain or take care of their grandchildren as the mothers of the children (in many cases single mothers) look for their livelihoods. The latter assertion is also strengthened by the fact that up to $70 \%$ of rotavirus infections in adults were among women. The finding of rotavirus among women could also point to transmission from children to adults (10).

Our findings concerning rotavirus infection in both children and adults, coupled with other studies which have mainly focused on the prevalence of the disease, indicate that the burden of rotavirus infection in both children and adults to public health cannot be underestimated. This high burden of infection in children, and availability of effective rotavirus vaccines $(14,15,18,23)$ to prevent childhood deaths and hospitalizations, underscores the need to introduce these vaccines in the national programs on immunization by African governments in order to save the lives of children. So far, 5 African countries have successfully introduced rotavirus vaccination in their vaccine schedules, with Kenya listed as one of those African countries eligible for GAVI (Global Alliance for Vaccine Initiative) in 2014 (26). Even as African governments prepare to roll out rotavirus vaccines within the national childhood immunization programs, evaluation of these vaccines post licensure remains critical. Of special importance is the continuous surveillance of the circulating strains, as this information will guide on policy decision making on the type of vaccine.

Three fundamental questions still remain in regard to introduction of these vaccinations in large scale in Africa: 1) what will be the serotype specific efficacy of these vaccines in regard to strain variation in Sub-Saharan Africa? 2) Would we expect an emergence of unusual strain or strains escaping vaccine protection? 3) Will significant increases in strain-related disease burden occur after the roll out of rotavirus vaccinations?

During this study, we found out that G1 [P8] and G9[P8] (which were both associated with long electropherotypes) were the most prevalent in Viwandani slum. A study by WHO African Rotavirus Surveillance Network (2) covering 8 countries showed that G1 and G2 were detected routinely. A closer look at their data also reveals that in Kenya, G1 to G9 were detected with G1[P8] accounting for the highest (23\%) followed by G8[P6] (15\%) and G9[P8] (8\%) of the top 3 strains. The finding of $\mathrm{G} 1[\mathrm{P} 8]$ as the most 
prevalent in Viwandani indicates minimum reassortment of the virus within this population, and any vaccine targeting the major circulating strains in Kenya would be beneficial to this community. In this study, however, we could not detect G2 and G8 strains-an indication of a possible strain variation within different regions in the country. Just as Mwenda and colleagues found out, we also found mixed genotypes and a significant number (7\% for G-type and $10 \%$ for Ptype) could not be typed among the children. The presence of untypeable strains could point towards the existence of non-human rotavirus strains in circulation (27). Alternatively, the results could point towards the importance of revising the common primers currently used in RT-PCR during human rotavirus typing.

Two vaccines, a monovalent vaccine-Rotarix (Glaxosmithkline) and a pentavalent vaccineRotateq (Merck \& Co Inc.), have been licensed. Rotarix as it is, provides cross protection to G1, G3, G4 and G9 whereas Rotateq provides cross protection to G1, G2, G3 and G4 viruses (28). It is therefore notable that the question of whether vaccine providing cross protection against all strain variants in Africa still remains pertinent. Although these vaccines have shown high efficacy in the western world and Latin America, a reduced efficacy has been reported in African and Asia countries. Further, 3 longitudinal strain surveillance studies done before and after routine childhood vaccinations have shown an upsurge of different strains. In their study, Carvalho-Costa and colleagues (29) noted a predominance of G2[P4] strain within 2 years after the introduction of a single strain vaccine in Brazil. On the other hand, Hull and colleagues noted an upsurge in G3[P8] strains after the introduction of a pentavalent vaccine (30). Further, a study done in Australia using the two types of vaccines (31) showed a higher prevalence of G2[P4] in the states that exclusively used the monovalent vaccine as opposed to G3[P8] strain that had a high prevalence in the states that used a pentavalent vaccine. Although the authors are cautious and warn against any conclusion with regard to the role of vaccination in the upsurge of the observed strains, their findings underscore the need for continuous strain surveillance and epidemiological studies prior to and post vaccine introduction for mass vaccination, especially in Africa where strain diversity remains a major concern.

A number of limitations were observed in our study, namely, lack of CD4 or HIV viral load in adults to conclusively link rotavirus infection to HIV infection. Further, we are unable to show whether there was any relationship between the adults in our study and the children; hence, the study cannot completely justify transmission within the groups. The study was also done within a small slum in Nairobi's urban setting and hence the findings cannot be extrapolated to cover strain diversity in the entire country.

In conclusion, our study shows the existence of strain diversity between adults and children circulating in Viwandani slums and that these strains would potentially be covered by the 2 vaccines recommended for rotavirus prevention in children. Our results have also shown the prevalence in HIV infected adults. We further recommend that continuous rotavirus strain surveillance be carried out in other slums in Nairobi, and the focus should also include HIV positive adults, as this will help elucidate the risk of children-to-adult or adult-to-children transmission. The data generated in future studies would also present a picture of whether indirect protection of adults would be feasible with the vaccination of children.

\section{ACKNOWLEDGEMENT}

We acknowledge Dr Sam Kariuki (Head of the Center for Microbiology KEMRI) and Dr Kioko of Mareba City Council Hospital for their assistance in collection of patient samples. Finally, we wish to thank the Kenya Medical Research Institute (KEMRI) and the Jomo Kenyatta University (College of Health Sciences) for authorizing this research.

\section{REFERENCES}

1. Parashar UD, Gibson CJ, Bresee JS, Glass RI. Rotavirus and severe childhood diarrhea. Emerging infectious diseases 2006; 12(2): 304-306.

2. Mwenda JM, Ntoto KM, Abebe A, et al. Burden and epidemiology of rotavirus diarrhea in selected African countries: preliminary results from the African Rotavirus 
Surveillance Network. The Journal of infectious diseases 2010; 202 (Suppl): S5S11.

3. Global networks for surveillance of rotavirus gastroenteritis, 2001-200. Releve epidemiologique hebdomadairel Section d'hygiene du Secretariat de la Societe des Nations = Weekly epidemiological record / Health Section of the Secretariat of the League of Nations, 2008; 83(47): 421-5.

4. Nokes DJ, Abwao J, Pamba A, et al. Incidence and clinical characteristics of group A rotavirus infections among children admitted to hospital in Kilifi, Kenya. PLoS medicine, 2008; 5(7): e153.

5. Kiulia NM, Peenze I, Dewar J, et al. Molecular characterisation of the rotavirus strains prevalent in Maua, Meru North, Kenya. East African medical journal, 2006; 83(7): 360-5.

6. Cox MJ, Medley GF. Serological survey of anti-group A rotavirus IgM in UK adults. Epidemiology and infection, 2003; 131(1): 719-26.

7. del Refugio González-Losa Ma, PolancoMarín GG, Manzano-Cabrera L, Puerto-Solís $\mathrm{Mn}$. Acute Gastroenteritis Associated with Rotavirus in Adults. Archives of medical research, 2001; 32(2): 164-7.

8. Nakajima H, Nakagomi T, Kamisawa T, et al. Winter seasonality and rotavirus diarrhoea in adults. Lancet, 2001; 357(9272): 1950.

9. Oyofo BA, Subekti D, Tjaniadi P, et al. Enteropathogens associated with acute diarrhea in community and hospital patients in Jakarta, Indonesia. FEMS immunology and medical microbiology 2002; 34(2): 139-46.

10. Anderson EJ, Weber SG. Rotavirus infection in adults. The Lancet infectious diseases 2004; 4(2): 91-9.

11. Wenman WM, Hinde D, Feltham S, Gurwith M. Rotavirus infection in adults. Results of a prospective family study. The New England journal of medicine, 1979; 301(6): 303-6.

12. Grimwood K, Abbott GD, Fergusson DM, Jennings LC, Allan JM. Spread of rotavirus within families: a community based study. British medical journal, 1983; 287(6392): 575-577.

13. Kim HW, Brandt CD, Kapikian AZ, et al. Human reovirus-like agent infection.
Occurrence in adult contacts of pediatric patients with gastroenteritis. JAMA: the journal of the American Medical Association, 1977; 238(5): 404-407.

14. Seheri LM, Page NA, Mawela MPB, Mphahlele MJ, Steele AD. Rotavirus vaccination within the South African Expanded Programme on Immunisation. Vaccine, 2012; 30 (Supplement 3): C14-C20.

15. Meeting of the Strategic Advisory Group of Experts on immunization, October 2009 conclusions and recommendations. Releve epidemiologique hebdomadairel Section d'hygiene du Secretariat de la Societe des Nations = Weekly epidemiological record/ Health Section of the Secretariat of the League of Nations 2009; 84(50): 517-32.

16. Lopman BA, Curns AT, Yen C, Parashar UD. Infant rotavirus vaccination may provide indirect protection to older children and adults in the United States. The Journal of infectious diseases, 2011; 204(7): 980-986.

17. Vesikari T, Matson DO, Dennehy $P$, et al. Safety and efficacy of a pentavalent humanbovine (WC3) reassortant rotavirus vaccine. The New England journal of medicine, 2006; 354(1): 23-33.

18. Vesikari $\mathrm{T}$, Itzler $\mathrm{R}$, Matson $\mathrm{DO}$, et al. Efficacy of a pentavalent rotavirus vaccine in reducing rotavirus-associated health care utilization across three regions (11 countries). International journal of infectious diseases : IJID : official publication of the International Society for Infectious Diseases, 2007; 11 Suppl 2: S29-35.

19. Ruiz-Palacios GM, Perez-Schael I, Velazquez FR, et al. Safety and efficacy of an attenuated vaccine against severe rotavirus gastroenteritis. The New England journal of medicine, 2006; 354(1): 11-22.

20. Linhares AC, Velazquez FR, Perez-Schael I, et al. Efficacy and safety of an oral live attenuated human rotavirus vaccine against rotavirus gastroenteritis during the first 2 years of life in Latin American infants: a randomised, double-blind, placebo-controlled phase III study. Lancet, 2008; 371(9619): 1181-1189.

21. Zaman K, Dang DA, Victor JC, et al. Efficacy of pentavalent rotavirus vaccine against severe rotavirus gastroenteritis in infants in 
developing countries in Asia: a randomised, double-blind, placebo-controlled trial. Lancet, 2010; 376(9741): 615-623.

22. Madhi SA, Cunliffe NA, Steele D, et al. Effect of human rotavirus vaccine on severe diarrhea in African infants. The New England journal of medicine, 2010; 362(4): 289-298.

23. Armah GE, Sow SO, Breiman RF, et al. Efficacy of pentavalent rotavirus vaccine against severe rotavirus gastroenteritis in infants in developing countries in sub-Saharan Africa: a randomised, double-blind, placebocontrolled trial. Lancet, 2010; 376(9741): 606614.

24. Gouvea V, Glass RI, Woods P, et al. Polymerase chain reaction amplification and typing of rotavirus nucleic acid from stool specimens. Journal of clinical microbiology, 1990; 28(2): 276-282.

25. Temu A, Kamugisha E, Mwizamholya DL, Hokororo A, Seni J, Mshana SE. Prevalence and factors associated with Group A rotavirus infection among children with acute diarrhea in Mwanza, Tanzania. Journal of infection in developing countries, 2012; 6(6): 508-815.

26. GAVI Alliance. List of countries eligible for GAVI support. 2014.

27. Banyai K, Bogdan A, Domonkos G, et al. Genetic diversity and zoonotic potential of human rotavirus strains, 2003-2006, Hungary. Journal of medical virology, 2009; 81(2): 362670.

28. van Hoek AJ, Ngama M, Ismail A, et al. A cost effectiveness and capacity analysis for the introduction of universal rotavirus vaccination in Kenya: comparison between Rotarix and RotaTeq vaccines. PloS one, 2012; 7(10): e47511.

29. Carvalho-Costa FA, Volotao Ede M, de Assis $\mathrm{RM}$, et al. Laboratory-based rotavirus surveillance during the introduction of a vaccination program, Brazil, 2005-2009. The Pediatric infectious disease journal, 2011; 30(1 Suppl): S35-41.

30. Hull JJ, Teel EN, Kerin TK, et al. United States rotavirus strain surveillance from 2005 to 2008: genotype prevalence before and after vaccine introduction. The Pediatric infectious disease journal, 2011; 30(1 Suppl): S42-47.

31. Kirkwood CD, Boniface K, Barnes GL, Bishop RF. Distribution of rotavirus genotypes after introduction of rotavirus vaccines, Rotarix $(R)$ and $\operatorname{RotaTeq}(\mathrm{R})$, into the National Immunization Program of Australia. The Pediatric infectious disease journal, 2011; 30(1 Suppl): S48-53. 